\title{
Gunshot (Pellets) injury of the Breast: A case report
}

\author{
${ }^{1}$ V I Nwagbara, ${ }^{1 *}$ Maurice E Asuquo, ${ }^{2}$ Cyril Agbor, ${ }^{2}$ Ijeoma E Nwachukwu \\ ${ }^{I}$ Department of Surgery, University of Calabar/University of Calabar Teaching Hospital, Calabar, Nigeria \\ ${ }^{2}$ Department of Surgery, University of Calabar Teaching Hospital, Calabar, Nigeria \\ *Corresponding authorE-mail: mauefas@yahoo.com,mauefas54@gmail.com
}

\begin{abstract}
Gunshot injuries form a major part of trauma but isolated breast injury due to gunshot is rare. Presented is a 40-year-old female with gunshot (pellets) injury of the breast. Initial surgeries to extract pellets resulted in partial amputation of the breast with surgical site infection. Management of this patient presented a dilemma in judgment of treatment in view of the effects of foreign bodies in the breast.
\end{abstract}

\section{Introduction}

Gunshot wounds (GSW's) constitutes a major part of trauma injuries, however, breast lesions due to GSW are uncommon [1]. The severity of injury depends on the type of firearm, velocity of the projectile, distance of shot from patient, and tissue resistance [2]. The large number of pellets dispersed in the tissues increase the damage with the attendant risk of infection. On the contrary, the presence of pellets may not have any consequence and be left in situ without any intervention [2,3]. Management of patients with shotgun wounds are controversial, clinical evaluation remain the most reliable indication to identify patients for emergency operation after shotgun wounds [2, 4]. Foreign body (FB) granuloma from previous GSW mimicking malignant breast mass has been reported [5]. We present this case to highlight the isolated breast injury due to pellets, challenges of clinical judgment to direct treatment in view of the injured organ and the type of injury.

\section{Case report}

A case report of a 40-year-old female who was first seen at the accident and emergency unit of the University of Calabar Teaching Hospital (UCTH), Calabar with a history of gun shot injury to the right breast of two weeks duration. She had been shot on her chest by armed bandits while walking home at night in Calabar. Following the shots, she noticed sharp pains over her right breast and chest; there was no difficulty breathing or loss of consciousness. She was not certain of the type of gun she was shot with.

She was rushed to a private hospital where she had two surgeries to explore the breast after a chest x-ray that showed pellets in the right breast (Figure 1). Ten and sixteen pellets were extracted in the first and second surgeries respectively as reported. On breakdown of the wound one week after the second surgery, she presented at UCTH, Calabar.

Examination revealed a pendulous breast with a transverse dehiscent wound extending along the entire width of the upper limit of the breast, wound edges were gaping, the floor covered with yellowish white necrotic slough and unhealthy granulation tissue and a seropurulent discharge, absorbable sutures were also found within, the surrounding skin was hyperemic with peau d'orange and tender.

Investigations done included: haematocrit: $35 \%$; chest x-ray revealed post extraction two round radiopacity in keeping with pellets in the region below the wound of the right breast, (Figure 2), wound swab microscopy and culture yielded Staphylococcus aureus sensitive to levofloxacin, gentamycin and sparfloxacin.

Diagnosis was a partial amputation of the right breast with surgical site infection.

Daily dressing of the wound with honey was commenced and antibiotic therapy with levofloxacin. The floor of the wound gradually filled with healthy granulation tissue and the wound contracted, (Figure 3). She had secondary wound closure and discharged to the surgical out patient department after removal of sutures. 
Follow up at the Surgical out patients was satisfactory with the wound healed.

\section{Discussion}

Gunshot wounds of the breast occur together with intra-abdominal injury at the rate of $50 \%$ but less frequently with chest wall and lung wounds [6]. Shotguns are capable of causing a wide variety of injuries depending on the weaponsvictim distance and the type of gun and pellets [4]. We report an isolated gunshot (pellets) breast injury in a 40-year-old female. Associated abdominal injury was unlikely in our patient as the target of the shot was the superior part of the breast, in addition the pellets did not penetrate the chest wall (Figure 1), perhaps due to the distance of the shot and the resistance of the breast tissue not to be underestimated. Pramod et al reported a GSW in the breast with silicone implant with thoracic trauma [7].

Basic firearm injuries are classified into three groups: penetrating as was our case, perforating and avulsion. Low velocity projectiles can result in penetrating wounds that create small entrance as depicted by the case presented and exit wound that was not the case presented [2]. In order to guide treatment and predict outcome, the number of body areas (Head and neck, chest, abdomen and the extremeties) injured classifies wounds. Grade I - more than 2 areas, Grade II - 2 adjacent areas and Grade III - one area [4]. The index patient suffered a Grade III injury. However, despite this classification, clinical examination had been shown to be the most reliable tool to identify patients for emergency operation [4]. Pellets scatter as they travel, the type of shot related to size, shape (round or irregular fragments) and weight of pellets correlate with morbidity and mortality [8]. Based on clinical evaluation, there was no indication for emergency surgery offered the patient. It stands also to reason that delay in extraction until when fibrosis had developed will engender difficulty in extraction; however, fibrous encasement may be protective and render the pellets inert.

Definitive management of patients with shotgun (pellets) penetrating wounds is controversial [2]. Isa Kara M et al [2] had reported shotgun wounds with many penetrated pellets of 26 years without symptoms. In another report viewing FB with potential of poisoning, recurrent infections and secondary haemorrhage recommended extraction even in the absence of clinical symptoms. The morbidity associated with our patient from multiple explorations to extract pellets resulted in partial amputation of the breast and wound infection in a patient that was clinically stable may not have been the best judgment at the time.

Metallic FB's in breast mammography had been reported from many sources including GSW's [9]. Granuloma from FB contains Langhans cells or FB giant-type giant cells. These cells emanate from epitheloid cells that aggregate and accumulate around poorly digestible materials [5]. There lies the challenge with soft tissue mass with multiple micro calcifications mimicking lesions suspicious of malignancy [5]. This may constitute an indication for extraction of pellets in the breast tissue. This is further highlighted as models of epithelial cells generation indicate that a causal link exist between physical trauma and cancer [10].

This case presents a dilemma in decision and a subject of debate. Extraction of pellets dispersed in a clinically stable patient may not be desirable in view of the deleterious effect of surgery as depicted in this case (partial amputation of the breast). However, the target organ such as the breast with a high risk of malignancy calls for long term follow up noting the possible challenges of imaging and diagnosis if associated with malignancy.

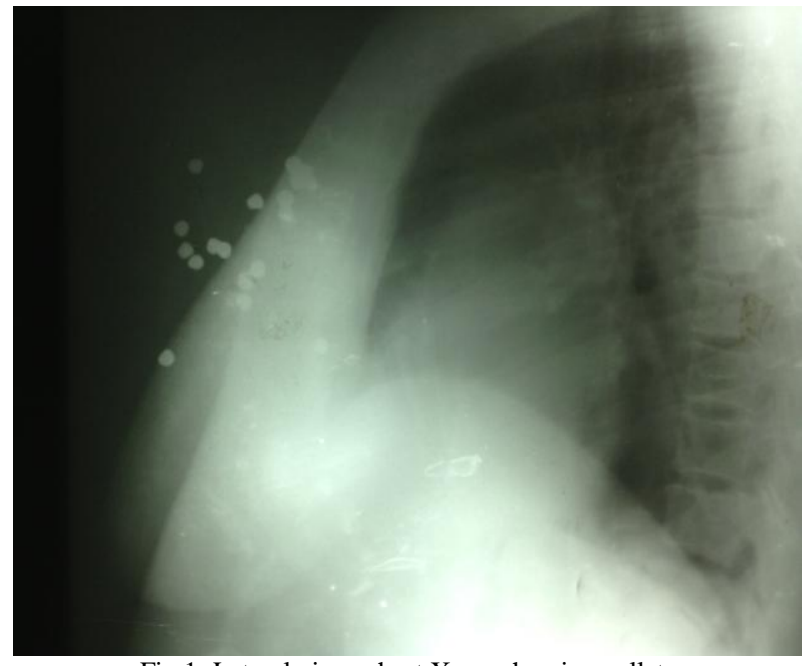

Fig.1: Lateral view, chest X-ray showing pellets

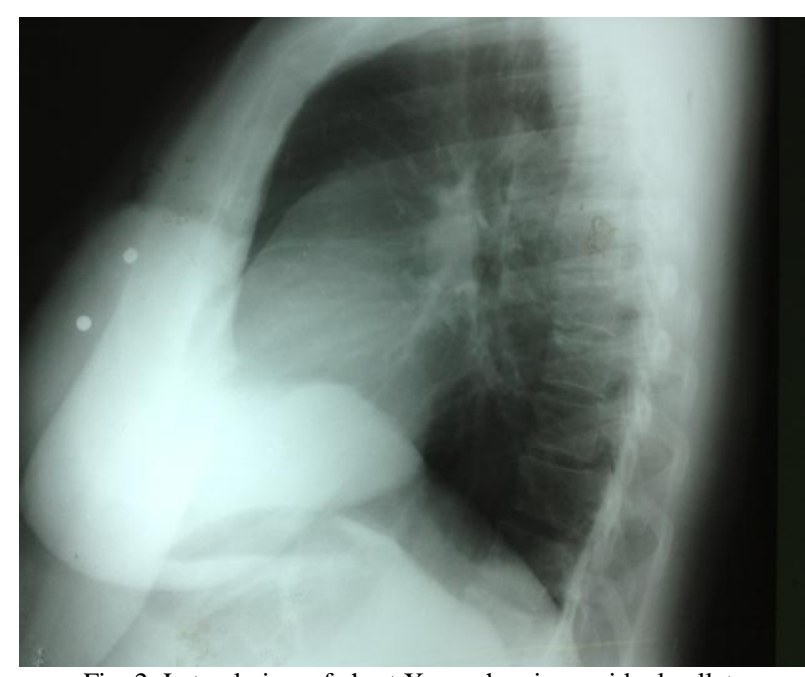

Fig. 2: Lateral view of chest $\mathrm{X}$-ray showing residual pellets 


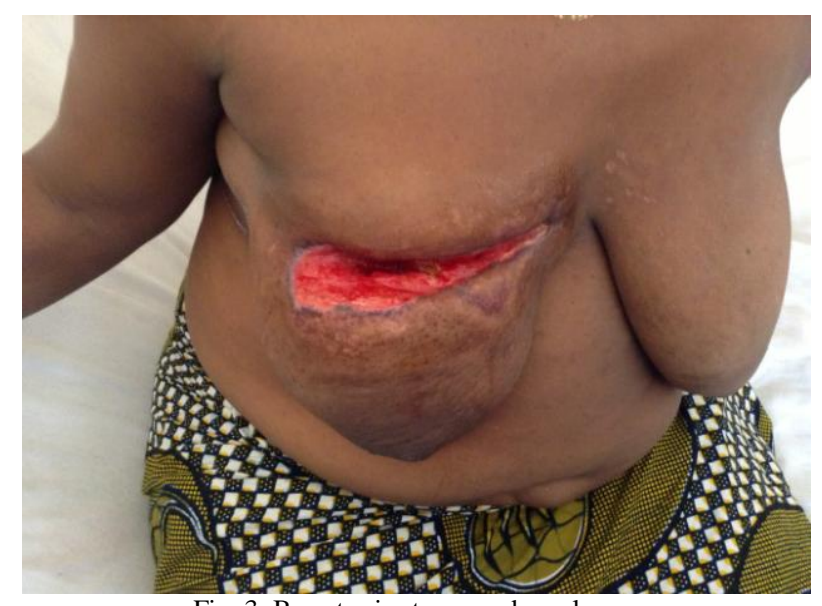

Fig. 3: Breast prior to secondary closure

\section{References}

[1] Onder A, Kapan M, Girgin S, Ankanoglu Z, Taskesen F, Beyazit O. Isolated breast trauma due to gunshot injury: case report. JAEM 2011, doi $: 10.5152 /$ jaem.2011.073.

[2] Isa Kara M, Polat HB, Ay S. Penetrated shotgun pellets: A case report. Eur J Dentistry 2 (2008) 51-62.

[3] Ullah O, Hassan T, Khan TM. Gunshot injury of the chest. JAPMI 4(1) (1990) 128-138.

[4] Velmahos GC,Safaoui M, Demetriades D. Management of shotgun wounds : do we need classification systems ? Int Surg 84(2) (1999) 99104.

[5] Wakabayashi M, Reid JD, Bhattacharjee M. Foreign body granuloma caused by prior gunshot wound mimicking malignant breast mass. AJR 173 (1999) 321-322.

[6] Renz BM, Hanzlick R. Gunshot wounds of the female breast: risk for intra-abdominal injury. South Med J 85 (1992) 1072-1076

[7] Pramod Nk, Thoma A. Breast implant rupture due to gunshot injury. Plast Reconst Surg 94 (1994) 893-894.

[8] Ordog GJ, Wasserberger J, Balasubramaniam S. Shotgun wound ballistics. J Traum 28(5) (1988) 624-632.

[9] Bassett LW, Jackson VP. Diagnosis of disease of the breast. Philadelphia. Saunders (1997) 378-382.

[10] Rigby JE, Morris JA, Lavelle J, Stewart M, Gatrell AC. Can physical trauma cause breast cancer? Eur J Cancer Prev 11(3) $2002307-311$. 